\title{
An enhanced Lemaitre model formulation for materials processing damage computation
}

\author{
Bouchard P.O., Bourgeon L., Fayolle S. and Mocellin K. \\ Mines ParisTech, CEMEF - Centre de Mise en Forme des Matériaux, CNRS UMR 7635, BP \\ 207, 1 rue Claude Daunesse, 06904 Sophia Antipolis Cedex, France
}

Corresponding author: Pierre-Olivier Bouchard

Email: pierre-olivier.bouchard@mines-paristech.fr

Tel: +33493678921

Fax: +33492389752

\begin{abstract}
The Lemaitre damage model is now widely used to deal with coupled damage analyses for various mechanical applications. In this article, different extensions of the model are presented and discussed to deal with complex multiaxial configurations - such as multi-stages bulk forming processes. A specific treatment is done to account for compressive damage growth, and a stress triaxiality cut-off value is considered to avoid any damage evolution below a critical negative triaxiality. The damage potential is also modified to deal with highly ductile materials, and the plastic strain is split into a negative part and a positive part to differentiate damage growth for compressive states of stress and for tensile states of stress. Finally, an anisotropic damage approach based on the comparison between grain flow orientation and principal loading directions is defined. A combination of these extensions is achieved within a single Lemaitre formulation. Application on different examples show the robustness and accuracy of the model defined in this paper.
\end{abstract}

KEY WORDS: Lemaitre damage model - compressive damage - multiaxial loadings anisotropic damage - stress triaxiality - chevrons cracking.

\section{Introduction}

Many damage approaches have been presented to predict damage growth for different kind of industrial applications. Most of the time, these models predict a good location of the maximal value of damage [1, 2], but remain inaccurate concerning the prediction of the instant of crack initiation, in particular when dealing with multi-stages forming processes. Indeed, the study of damage growth in the context of materials forming is extremely complex. This is due to many reasons:

- Multiaxial loadings: during a manufacturing process, the material is subjected to complex strain paths, involving successively compression, tension or shearing. Damage mechanisms are clearly different for each of these specific loadings. It is thus difficult to get a unique damage model that can predict accurately damage growth for each of these mechanical states.

- Non-monotonic loadings: another particular aspect of forming processes is that the material is subjected to nonmonotonic loadings. This means that voids that where created in tension can then be subjected to compressive loading, involving crack closure effects.

- Simple parameters identification: whatever the damage model used, the identification of damage parameters is an important issue. Most of the time this identification is performed on simple tensile mechanical tests. This means that damage parameters are identified for a uniaxial monotonic loading, which is not adapted to what has been described in the two first points. Moreover, for anisotropic materials, one single mechanical test does not allow identifying the anisotropic behaviour [3, 4].

- Numerical dependencies: to model accurately the softening part of the material behaviour, damage has to be coupled with the behaviour law of the material. It is well-known now that this coupling gives rise to the phenomenon of damage localization, which means that damage evolution depends on the mesh size. Several non local approaches have been defined in the last decade [5-8]. However these models often introduce a material 
characteristic length which remains difficult to calibrate. In addition, the physical meaning of this characteristic length is still not clearly defined for many materials.

For seek of simplicity, uncoupled damage models are still widely used in the industry. The damage parameter is computed using an integral of a strain and stress function, and its distribution can be studied in a post-processing step. Among the numerous existing models, we can quote the models introduced by: Cockcroft \& Latham [9] which is based on the maximal principal stress, Mc Clintock [10] which also includes the minimal principal stress and the hardening parameter, Rice \& Tracey [11] based on the triaxiality rate or Oyane and co-workers [12] which comes from the plasticity approach of porous materials. More recently, Bai and Wierzbicki have presented a new formulation accounting for the Lode dependence on fracture [13]. Uncoupled models are easy to implement in numerical software, but are quite unrealistic because the damage evolution does not influence the material properties.

Using a coupled approach, damage and mechanical properties are directly related and the material fracture process is modeled by a progressive decrease of the global response of the structure. Some of these models [14-17] use the notion of effective stress which represents the actual stress transmitted by the bulk material between the microdefects. Another frequently used approach consists in introducing a damage variable $f_{v}$ which represents the volumetric fraction of voids in the material. The parameter $f_{v}$ is then used in the material constitutive law and interacts with the others state variables. The damage model of Tvergaard-Needleman [18], based on the model introduced by Gurson [19] belongs to these approaches. More recently, a new model accounting for the Lode angle has been introduced by Xue and coupled with the material behaviour to describe the material deterioration [20]. Contrary to uncoupled approaches, coupled damage models are more difficult to implement in numerical software. However these models are closer to the physical damage phenomenon and give a better representation of the progressive damage evolution within the material and how it progressively leads to fracture.

Numerical modelling applied to metal forming processes has given rise to many studies during these last ten years [21-23]. The use of coupled damage approaches within the framework of metal forming is more recent [1, 2, 24-26].

In this paper, the phenomenological Lemaitre damage approach is considered [27]. Several modifications of the model are presented to improve its predictivity when dealing with multi-stages forming processes. Some of these modifications come from the literature (crack closure effects [14], cut-off value for triaxiality [28]), and others are defined here to improve damage prediction (new damage potential, anisotropic damage methodology). The grain flow orientation is also computed during the manufacturing process in order to account for anisotropic damage.

In the first part, some specificities of metal forming processes are shown regarding stress triaxiality evolution. Then the isotropic "classical" Lemaitre damage model is presented in the second part, and details are given concerning its implementation within a velocity-pressure formulation. In the third part, different improvements of the model are described to enrich its initial formulation. Particular attention is paid to damage evolution in compression, highly ductile materials and damage anisotropy. Finally the fourth part illustrates, through different examples, some improvements in terms of damage prediction.

\section{Forming processes}

In numerous cold forming processes, the material is subjected to different forming steps to reach the final shape of the industrial part.

Fig. 1.a presents the final shape of the three stages required to obtain a speed transmission shaft. At the end of the process, the circumferential part of the transmission shaft head may contain some microdefects or microcracks depending on the ductility of the steel grade used.

Fig. 1.b shows the evolution of stress triaxiality at the critical position during the three last stages. Stress triaxiality is the ratio between hydrostatic pressure and equivalent von Mises stress. It is one of the most important parameter when dealing with damage growth since positive triaxiality means global tension applied to the material.

We can see that the material is subjected to compression (negative stress triaxiality) during a long period before switching to tensile loadings and potential crack initiation during the last stage. At the end of the process, Fig. 1.b shows that the stress triaxiality field is positive. 


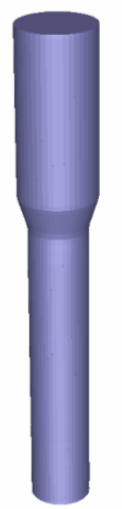

End of stage 1

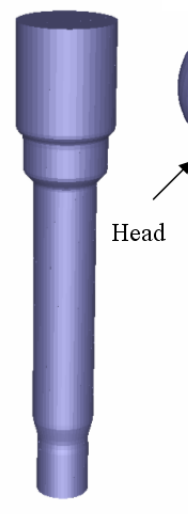

End of stage 2

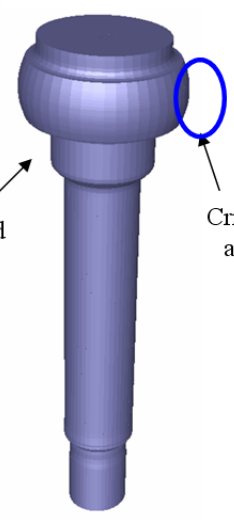

End of stage 3

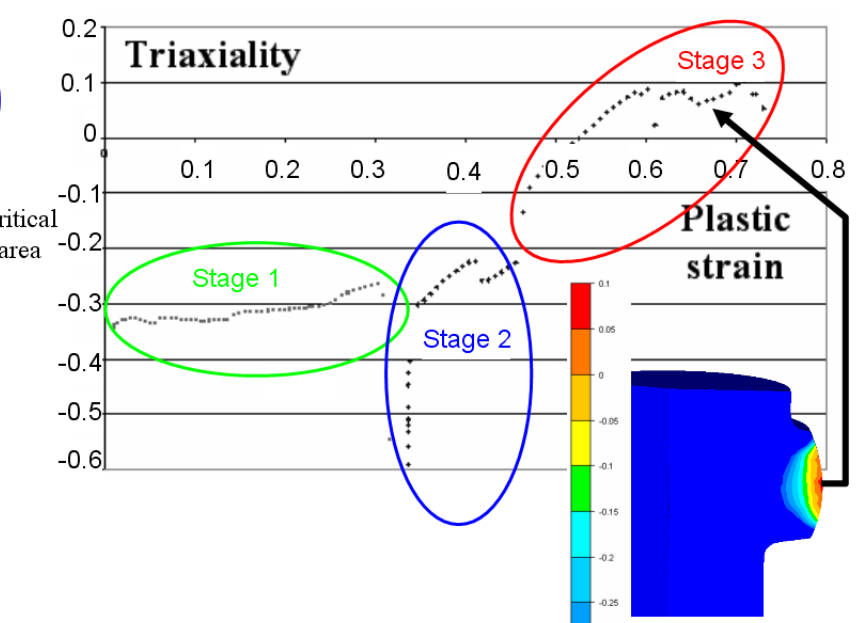

Stress

triaxiality

field

a)

b)

Figure 1. a) Cold forging of a speed transmission shaft - b) Triaxiality path during the three last stages of the forming process

For such cold forming operations, the initial cylindrical billet contains elongated inclusions oriented towards the axis of the billet. These elongated inclusions come from the rolling process used to produce these cylindrical bars. It has been shown in [29] that such materials can exhibit a strong anisotropy depending on the direction of inclusions. It is also interesting to point out the fact that on the collar, maximal principal stresses are oriented towards the circumferential direction. This means that principal stresses are perpendicular to the inclusions orientations. This must be accounted for within the damage model and the damage parameters identification.

This example is quite representative of most cold forging processes. At the beginning, the initial billet is often subjected to compressive loadings. Then the critical areas of the final geometry of the part are the ones that undergo high positive stress triaxiality.

\section{Coupled Lemaitre damage approach}

\section{Lemaitre Damage Model}

This ductile damage model is based on a thermodynamic framework [14]. In its scalar form, damage is represented by the state internal variable $D(0 \leq D<1)$ which represents the ratio of damaged area of a unit surface $S_{D}$ divided by the total surface $S: D=S_{D} / S$.

Based on the strain equivalence hypothesis, the effective stress tensor $\tilde{\sigma}$ is defined as the tensor that actually resists to the load:

$$
\tilde{\sigma}=\frac{\sigma}{1-D}
$$

where $\sigma$ is the stress tensor for the undamaged material.

The damage strain energy release rate $Y$ is associated to the damage variable $D$. It is given by:

$$
-Y=\frac{q^{2}}{2 E(1-D)^{2}}\left[\frac{2}{3}(1+v)+3(1-2 v)\left(\frac{\sigma_{H}}{q}\right)^{2}\right]
$$


where $q=\sqrt{\frac{3}{2} s: s}$ is the von Mises equivalent stress, and $s$ is the deviatoric stress part. $E$ is the Young Modulus, $v$ the Poisson ratio, $\sigma_{H}$ the hydrostatic stress and $\frac{\sigma_{H}}{q}$ defines the stress triaxiality ratio. This last parameter plays an important role on damage evolution. The evolution of internal variables can be obtained via normality rule by supposing the existence of a plasticity/damage dissipation potential $F$, which is a convex function of associated variables. This potential is the sum of $f$ and $F_{Y}$ that are respectively the von Mises plasticity criterion and the Lemaitre potential of dissipation related to damage:

$$
F=f+F_{Y} \text { with }\left\{\begin{array}{c}
f \boldsymbol{\sigma}, R=\tilde{q}-R-\sigma_{Y} \\
F_{Y}=\frac{S_{0}}{b+1} \frac{1}{1-D}\left(\frac{-Y}{S_{0}}\right)^{b+1}
\end{array}\right.
$$

where $S_{0}$ and $b$ are materials parameters, $R$ is the scalar variable associated with isotropic hardening and $\sigma_{Y}$ is the yield stress. $\tilde{q}$ is the equivalent effective stress tensor that can be defined by:

$$
\tilde{q}=\sqrt{\frac{3}{2}\left(\frac{s}{1-D}\right):\left(\frac{s}{1-D}\right)}
$$

A kinematic hardening parameter can be added to the von Mises plasticity criterion if necessary.

Damage evolution is then given by the following relationship:

$$
\dot{D}=-\dot{\lambda} \frac{\partial F_{Y}}{\partial Y}
$$

where $\dot{\lambda}$ is the plastic multiplier.

Using (3) and (5) it follows that

$$
\dot{D}=\left(\frac{-Y}{S_{0}}\right)^{b} \dot{\bar{\varepsilon}}^{p}
$$

where $\dot{\bar{\varepsilon}}^{p}$ stands for the equivalent plastic strain rate and $\dot{\bar{\varepsilon}}^{p}=\sqrt{\frac{2}{3} \dot{\varepsilon}^{p}: \dot{\varepsilon}^{p}}$.

Damage increases only once the equivalent plastic strain has exceeded a strain threshold $\varepsilon_{D}$ :

$$
\dot{D}= \begin{cases}0 & \text { if } \bar{\varepsilon}_{p}<\varepsilon_{D} \\ \left(-\frac{Y}{S_{0}}\right)^{b} \dot{\bar{\varepsilon}}_{p} & \text { if } \bar{\varepsilon}_{p} \geq \varepsilon_{D}\end{cases}
$$

Finally, a critical damage value $D_{c}$ is used to account for macroscopic fracture. When the damage variable $D$ reaches $D_{c}, D$ is immediately set to 1 in order to model fracture. 


\section{Numerical implementation}

We use here the finite element software FORGE2005® that can handle 2D axisymmetric/plane strain as well as $3 \mathrm{D}$ configurations.

FORGE2005® is based on a mixed velocity-pressure formulation and the so-called Mini-element $(\mathrm{P} 1+/ \mathrm{P} 1)$ is used [30]. It is based on linear isoparametric tetrahedra (triangles for 2D configurations) and a bubble function is added to the linear velocity field at the element level in order to satisfy the Brezzi/Babuska condition. The space discretization based on this element, associated to the incremental formulation of the virtual work principle, lead to a set of discretized non-linear equations. The well-known iterative Newton-Raphson linearization method is used.

Small strain approach can be used since an updated Lagrangian formulation is adopted. For small time steps, plastic strain remains sufficiently small to respect small strain hypothesis. A classical one step Euler scheme is used to update the geometry at time $\mathrm{t}+\Delta \mathrm{t}$ once the solution at time $\mathrm{t}$ is known.

Besides an automatic adaptive remesher, based on mesh topology improvement [31] enables to deal with large deformation without losing accuracy. This remeshing stage is important during forming simulation since the mesh gets distorted due to large deformations.

FORGE2005® has been developed to model large deformation of viscoplastic, elastic-plastic and elasticviscoplastic materials. The Lemaitre damage model, presented in paragraph 3.1 has been implemented to deal with damage evolution during the forming operations. A weak coupling and a strong coupling have been implemented to couple the evolution of damage with the material behaviour.

\section{Damage coupling}

Due to the presence of damage $D$ within the material, an effective deviatoric stress $\tilde{S}$ and an effective hydrostatic pressure $\tilde{p}$ are defined:

$$
\left\{\begin{array}{l}
\tilde{s}=\frac{s}{(1-D)}=\frac{2 \mu e^{e}}{(1-D)} \\
\tilde{p}=\frac{p}{(1-D)}=\frac{-\chi \cdot \operatorname{trace}\left(\varepsilon^{e}\right)}{(1-D)}
\end{array}\right.
$$

where $\mu$ and $\chi$ are respectively the shearing modulus and the bulk modulus and $\varepsilon^{e}$ and $e^{e}$ are respectively the elastic strain tensor and its deviatoric part.

Damage is then coupled with plasticity by replacing $s$ by $\tilde{S}$ in the von Mises criterion.

Using the normality rule, the deviatoric strain rate tensor $\dot{e}^{p}$, and the hardening ratio $\dot{r}$ are given by:

$$
\left\{\begin{array}{l}
\dot{e}^{p}=\dot{\lambda} \frac{\partial f}{\partial s}=\frac{3}{2} \frac{\dot{\lambda}}{(1-D)} \frac{s}{q} \\
\dot{r}=-\dot{\lambda} \frac{\partial f}{\partial R}=\dot{\lambda}
\end{array}\right.
$$

where $\dot{\lambda}$ is the plastic multiplier.

It is then possible to write the damage evolution by: 


$$
\dot{D}=\left(\frac{-Y}{S_{0}}\right)^{b} \dot{\bar{\varepsilon}}_{p}=\left(\frac{-Y}{S_{0}}\right)^{b} \frac{\dot{r}}{(1-D)}
$$

To account for the elastic plastic behaviour, an elastic predictor/plastic corrector with a radial return algorithm is used. The damage elastic plastic behaviour is solved by coupling a Prandtl-Reuss law to damage. So that for each time step $n+1$, we have to find the hardening increment $\Delta r$ and the new damage value $D_{n+1}$. These two unknowns are computed simultaneously at each iteration of a Newton-Raphson algorithm by solving the two following equations:

$$
\left\{\begin{array}{l}
f_{1}=\quad \tilde{q}_{n+1}^{T}-\frac{3 \mu \Delta r}{\left(1-D_{n+1}\right)}-R_{n+1}=0 \\
f_{2}=D_{n+1}-D_{n}-\left(\frac{-Y_{n+1}}{S_{0}}\right)^{b} \frac{\Delta r}{\left(1-D_{n+1}\right)}=0
\end{array}, \text { where } \tilde{q}_{n+1}^{T}=\sqrt{\frac{3}{2} \tilde{s}_{n+1}^{T}: \tilde{s}_{n+1}^{T}}\right.
$$

with $X^{T}$ means the trial value of the variable $X$.

This non linear system is linearized and solved using an iterative Newton-Raphson algorithm. Two types of coupling can be used:

- a strong coupling, in which both equations are solved simultaneously to obtain the two unknowns $\Delta r$ and $D_{n+1}$;

- a weak coupling, which consists in solving only the first equation of the system (Equation 11.1) relative to the plasticity criterion in which we assume that $D_{n+l}$ is equal to $D_{n}$, in order to obtain $\Delta r$. Then the second equation is solved to compute the new damage value $D_{n+1}$.

In practice, even if the strong coupling gives more accurate results, the weak coupling is generally preferred for computation time reasons in industrial case forming simulation.

\section{Damage localization}

Coupling damage with the material behaviour law leads to a softening of the material's behaviour. One of the consequences of this softening is a numerical dependency. A finer mesh will lead to a faster damage evolution meaning that the numerical solution depends on the mesh size. Several techniques have been defined to deal with this phenomenon by regularizing the solution [5-8]. An integral non local approach as well as an implicit gradient non local approach have been implemented in the software. If these non local approaches are not used, it is necessary to work with the same mesh size as the one used to identify damage parameters. More details about non local models can be found in [32].

\section{Extensions of the Lemaitre damage model}

During multi-stages forming processes, the material can undergo successively high or low compressive states, shearing as well as tensile mechanical loadings.

The classical scalar Lemaitre approach has to be modified:

- Compressive damage evolution: a specific treatment has to be done when dealing with compressive loadings. The so-called crack closure effect [14,33], as well as a negative triaxiality limit [28] have been introduced to deal with compressive damage. These two modifications have been published already. However, they have been published separately, and as it will be shown in the next section, the combination of these modifications enables to obtain more predictive results.

- Highly ductile materials: in some cases, it is not possible to identify the Lemaitre damage parameters to match experimental results. This is the case for the highly ductile materials studied in this work. The 
damage potential has been modified in order to fit experimental results.

- Damage anisotropy: in some cases, materials microstructure induces anisotropic damage properties. In particular, this is true for billets obtained using cold extrusion processes or cold forging. A simple methodology is presented here to deal with anisotropic damage behaviour.

\section{Compressive damage evolution}

It is not easy to clearly represent the evolution of damage in compressive areas. In its initial form, the strain energy release rate $\mathrm{Y}$ is a function of the square of triaxiality, which means that the evolution of damage would be the same whatever the sign of triaxiality.

However, from a physical point of view, we cannot consider that the damage increase will be the same for an equivalent level of compressive or tensile state.

To find a more physical solution, some authors consider that there is no damage evolution in compressive areas, so that $\dot{D}$ is activated only for a positive triaxiality. But this solution does not account for some particular aspects of microvoids or microcracks closure during compressive stages.

We present here two improvements that have been published in the literature and we discuss their respective contribution for the upsetting test of a tapered specimen.

\section{Crack closure effects}

In the Lemaitre damage approach and for a cut of the specimen, the effective surface $\tilde{S}$ is equal to the total surface $S$ minus the surface of the voids $S_{D}$. So that the effective stress $\tilde{\sigma}$ is the stress $\sigma$ brought back to the surface $\tilde{S}$ that actually resists to the load.

In its initial formulation, the Lemaitre damage model considers that the damage increase is the same in tension $\left(\sigma_{H}>0\right)$ and compression $\left(\sigma_{H}<0\right)$. However, a compressive state tends to close already existing microcracks or microvoids, thus leading to an increase of the load bearing area for compressive states. The previous assumption (Equation 1) is true for tensile stresses, whereas for compressive stresses, Lemaitre has suggested the use of a regularization variable $h$ to account for crack closure effects:

$$
\tilde{\sigma}=\frac{\sigma}{1-h D}
$$

where $h(0 \leq h \leq 1)$ is a material parameter accounting for crack closure effects. The value of $h$ is often taken close to 0.2 for steels [14].

For multiaxial stress states it is however more accurate to split the stress tensor into a positive part $\left(\sigma_{+}\right)$and a negative part $\left(\sigma_{-}\right)[34]$ :

$$
\begin{gathered}
\sigma=\sigma_{+}+\sigma_{-} \text {with } \sigma_{+}=\sum_{i=1}^{3}<\sigma_{i}>e_{i} \otimes e_{i} \text { and } \sigma_{-}=\sum_{i=1}^{3}<-\sigma_{i}>e_{i} \otimes e_{i} \\
\sigma_{i}: \sigma \text { eigen values } \\
e_{i}: \sigma \text { eigen vectors }
\end{gathered}
$$

where the $\left\langle x>\right.$ symbol denotes the positive part: $\quad<x>=\left\{\begin{array}{l}x \text { if } x \geq 0 \\ 0 \text { if } x<0\end{array}\right.$ 
Based on this stress decomposition, it is then possible to write the expression of damage evolution by modifying the strain energy release rate associated with damage:

$-Y=\frac{-1}{2 E\left(-D^{2}\right.} \mathbf{K}+v \bar{g}_{+}: \sigma_{+}-v<\operatorname{tr} \sigma>^{2}-\frac{h}{2 E\left(-h D_{-}^{2}\right.} \mathbf{K}+v \bar{g}_{-}: \sigma_{-}-v<-\operatorname{tr} \sigma>^{2}{ }_{-}^{-}(14)$

This method is more accurate since it allows dissociating tensile and compressive contributions to damage evolution.

\section{Cut-off value of stress triaxiality}

To study the influence of negative triaxiality on damage evolution, Bridgman [35] has performed tensile tests on specimen subjected to hydrostatic pressure. Bridgman results show that deformation at fracture increases with hydrostatic pressure resulting in a longer striction stage before fracture. More recent studies have also confirmed this phenomenon [36, 37].

Based on these experimental observations, Bao and Wierzbicki [28] have shown that there was a negative triaxiality limit below which there was no more damage evolution.

It can thus be considered that exceeding this $-1 / 3$ limit value is a necessary condition for damage to increase. Under $-1 / 3$, the damage growth rate is set equal to 0 .

$$
\dot{D}=\left\{\begin{array}{l}
0 \quad \text { if } \frac{\sigma_{h}}{q} \leq-1 / 3 \\
\left(-\frac{Y}{S_{0}}\right)^{b} \dot{\bar{\varepsilon}}_{p} \quad \text { if } \frac{\sigma_{h}}{q}>-1 / 3
\end{array}\right.
$$

\section{Modification of the damage potential}

Damage evolution is depending on the choice of the damage potential. The potential introduced by Lemaitre is the most used one. However, other formulations have been presented in the literature:

- Lemaitre [14]: $F_{Y}=\frac{S_{0}}{(b+1)(1-D)}\left(\frac{-Y}{S_{0}}\right)^{b+1}$

- Tai and Wang [38]: $F_{Y}=\frac{S_{0} \cdot D}{2}\left(\frac{-Y}{S_{0}}\right)^{2}$ 
- Chandrakanth and Pandey [39]: $F_{Y}=\frac{1}{2} \frac{S_{0}}{D^{\alpha / n}\left(\bar{\varepsilon}_{p}\right)^{\alpha / n}}\left(\frac{-Y}{S_{0}}\right)^{2}$

- Bonora [40]: $F_{Y}=\frac{1}{2} \frac{S_{0}\left(D_{c}-D\right)^{(\alpha-1) / \alpha}}{(1-D) \cdot\left(\bar{\varepsilon}_{p}\right)^{(2+n) / n}}\left(\frac{-Y}{S_{0}}\right)^{2}$

Parameters $S_{0}, D_{c}, b, \alpha$ and $n$ are damage materials parameters, and $Y$ is the damage strain energy release rate as defined in Equation 2. Depending on the choice of this damage potential, the damage increase will be higher or lower with respect to deformation. In some cases, such as for highly ductile materials, it is not possible to match experimental results using the classical Lemaitre potential. For such ductile materials, we modify the Lemaitre potential by adding a term depending on the equivalent plastic strain:

$$
F_{Y}=\frac{S_{0}}{b+1} \frac{1}{1-D}\left(\frac{-Y}{S_{0}}\right)^{b+1} \frac{1}{\left(\bar{\varepsilon}_{p}\right)^{\alpha}}
$$

where $\alpha$ is a new material damage parameter.

However, during bulk forming processes, the material is often successively subjected to compressive and tensile loadings. It is thus important here to dissociate the plastic strain that has been created in tension, and in compression. This idea is close to what is done by Pirondi and Bonora for ductile damage evolution for cyclic loadings [41].

Respectively tensile and compressive equivalent plastic strain are defined as follow:

- Tensile cumulative plastic strain :

$$
\begin{gathered}
\dot{\bar{\varepsilon}}_{p}^{+}=\left\{\begin{array}{cc}
0 & \text { if compression } \\
\dot{\bar{\varepsilon}}_{p} & \text { if tension }
\end{array} \text { and }\left.\quad \bar{\varepsilon}_{p}^{+}\right|_{n+1}=\left.\bar{\varepsilon}_{p}^{+}\right|_{n}+\dot{\bar{\varepsilon}}_{p}^{+} d t\right. \\
\dot{D}^{+}=\left\{\begin{array}{l}
0 \\
\left(-\frac{Y}{S_{0}}\right)^{b} \dot{\bar{\varepsilon}}_{p}^{+} \frac{1}{\left(\bar{\varepsilon}_{p}^{+}\right)^{\alpha}} \quad \text { if } \bar{\varepsilon}_{p}^{+}<\varepsilon_{D} \geq \varepsilon_{D}
\end{array}\right. \\
\left.D^{+}\right|_{n+1}=\left.D^{+}\right|_{n}+\dot{D}^{+} d t \quad \underline{\text { Damage due to tension }}
\end{gathered}
$$

- Compressive cumulative plastic strain :

$$
\dot{\bar{\varepsilon}}_{p}^{-}=\left\{\begin{array}{c}
0 \quad \text { if tension } \\
0 \quad \text { if compression and } \sigma_{h} / q \leq-1 / 3 \\
\dot{\bar{\varepsilon}}_{p} \quad \text { if compression and } \sigma_{h} / q>-1 / 3
\end{array} \text { and }\left.\bar{\varepsilon}_{p}^{-}\right|_{n+1}=\left.\bar{\varepsilon}_{p}{ }^{-}\right|_{n}+\dot{\bar{\varepsilon}}_{p}^{-} d t\right.
$$




$$
\begin{aligned}
\dot{D}^{-}= & \left\{\begin{array}{cr}
0 & \text { if } \bar{\varepsilon}_{p}^{-}<\varepsilon_{D} \\
\left(-\frac{Y}{S_{0}}\right)^{b} \dot{\bar{\varepsilon}}_{p}^{-} \frac{1}{\left(\bar{\varepsilon}_{p}^{-}\right)^{\alpha}} \text { if } \bar{\varepsilon}_{p}^{-} \geq \varepsilon_{D}
\end{array}\right. \\
\left.D^{-}\right|_{n+1}=\left.D^{-}\right|_{n}+\dot{D}^{-} d t & \text { Damage due to compression }
\end{aligned}
$$

Finally the total damage is:

$$
\left.D\right|_{n+1}=\left.D^{+}\right|_{n+1}+\left.D^{-}\right|_{n+1}
$$

In equations (21) and (24), it is assumed that tension (respectively compression) corresponds to a positive (respectively negative) value of the stress triaxiality ratio.

\section{Damage anisotropy}

Most of the time, damage is considered as isotropic for simplicity reasons. However, for cold forming processes, the initial steel billet usually comes from previous extrusion or rolling processes. These processes clearly give a preferential orientation to the material's microstructure: grains and inclusions are oriented in the rolling or extrusion direction [29]. If no thermal treatment is performed, important anisotropic behaviour can be observed at the forging step. It is thus important to identify critical damage values for different directions and to account for this damage anisotropy to predict fracture.

A 3D damage tensor would be necessary to deal accurately with non-monotonic multiaxial loadings. However, the identification of 3D damage tensor evolution laws can become extremely time consuming, in particular if damage growth is anisotropic. From an industrial point of view, it is often required to find faster methods. A solution based on the computation of the angle between the grain flow orientation and the direction of principal loading is presented here.

Fig. 2 shows the influence of the anisotropy that can be observed on ductile steel grades dedicated to cold forming processes. Three tensile specimens are cut from a cylindrical grade A steel billet: one in the longitudinal direction, one in the radial direction and the last one at $45^{\circ}$ with respect to the longitudinal direction. Fig. 2a shows that ductility is much more important in the longitudinal direction than in the radial direction [29]. This kind of anisotropic ductility often comes from the rolling process used to obtain cylindrical steel billets.

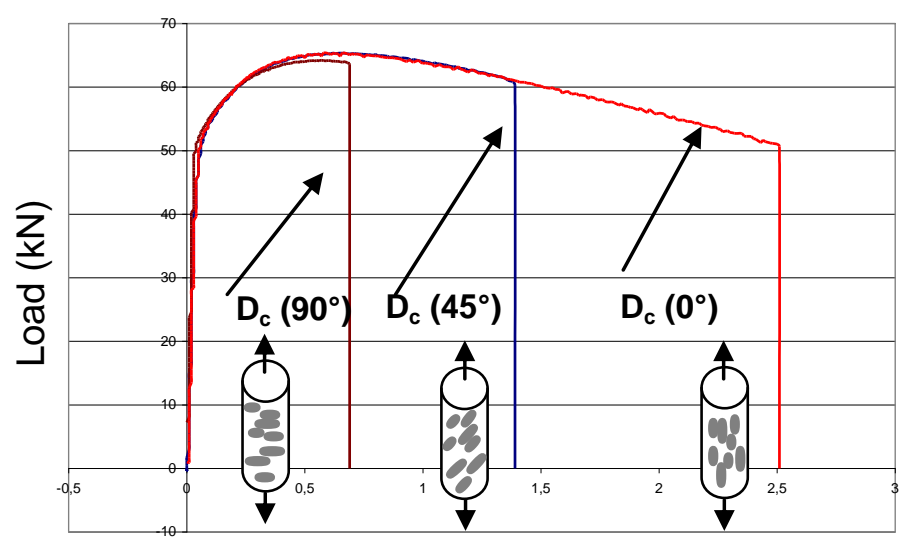

a)
Displacement

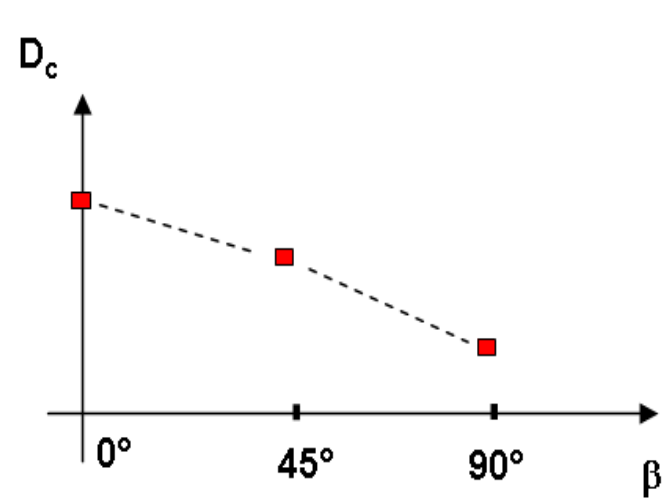

b)

Fig. 2. a) Tensile test on grade $A$ for three different specimens cut at $0^{\circ}, 45^{\circ}$ and $90^{\circ}$ with respect to the billet axis and b) identified critical damage values Dc for each direction, $\beta$ is the angle between the specimen axis and the kneading direction. 
It is interesting to see that damage seems to be anisotropic only in terms of critical damage value leading to fracture. Hardening and softening part of these curves are superimposed before fracture for the three specimens. From a phenomenological point of view, it can be assumed that $D_{c}$ is the only anisotropic damage parameter for such a material. It must be noted that from a microstructural point of view, this assumption is probably wrong. Indeed this anisotropic ductility may come from the highly oriented microstructure with grain flow orientation in the direction of the rolling process. In [29], the authors have shown the important role of particles orientation on damage mechanisms. However, the phenomenological approach presented here aims at improving damage prediction in a simple manner with a scalar damage approach.

In Fig. $2 \mathrm{~b}$, the evolution of the critical damage value $D_{c}$ is plotted with respect to the angle $\beta$ between the grain flow orientation and the loading direction. This evolution appears to be linear with respect to the angle $\beta$.

The use of such anisotropic model for a multiaxial cold forming process requires the computation of the grain flow orientation direction during the simulation. An initial grain flow orientation is entered at the beginning of the computation, and the evolution of this preferential orientation is computed during the simulation.

The deformation gradient $F$ is used. It is defined by:

$$
F_{i j}=\delta_{i j}+\frac{\partial u_{i}}{\partial X_{j}} \quad(i, j=1,3)
$$

where $u$ stands for the displacement field and $X$ for the material points coordinates.

If $d X_{n}$ is the kneading direction at increment $n$, then at increment $n+1$, the grain flow orientation is simply obtained by:

$$
d X_{n+1}=F \cdot d X_{n}
$$

Fig. 3 shows the numerical and experimental grain flow orientation obtained after the compression of a cylindrical billet with an initial vertical grain flow orientation.

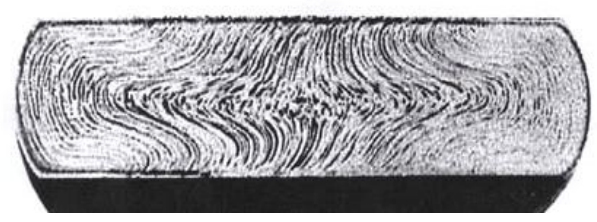

a)

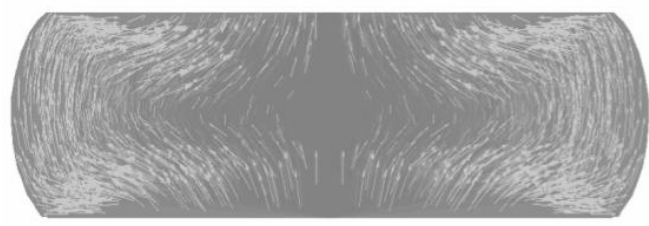

b)

Fig. 3. Experimental (a) and numerical (b) grain flow orientation after compression of a cylindrical billet

To account for anisotropy, it is necessary to compute at each increment the angle between the grain flow orientation and the loading direction. A diagonalization of the stress tensor is performed and the angles $\beta_{i}(\mathrm{i}=1,3)$ between each principal stress values and the grain flow orientation are computed (Fig. 4). 


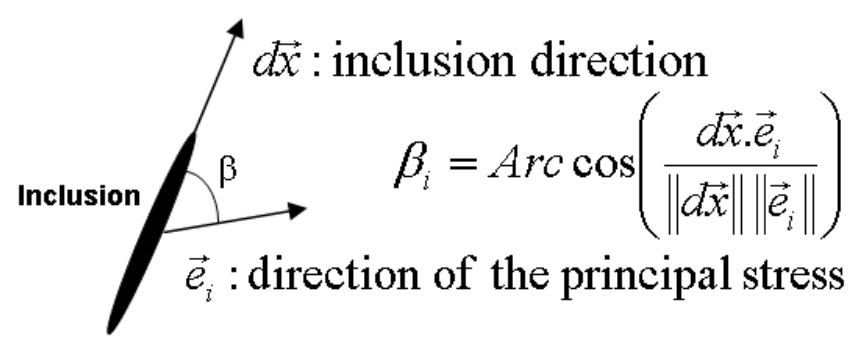

Fig. 4. Computation of the angle between inclusions direction and principal stresses

As shown in Fig. 2, it can be assumed here that the elastic-plastic behaviour and the damage growth are isotropic. This means that the damage parameters associated with damage evolution (i.e. $S_{0}, b, \varepsilon_{d}, h$ and $\alpha$ ) can remain unchanged. The only anisotropic variable to be considered is $D_{c}$ for this material. $D_{c}$ is a linear function of the angle between grain flow orientation and the direction of the maximal principal stress (Fig. 2b).

However it must be stressed out that this analysis of the material anisotropy can be done here for this material and within a phenomenological framework. Indeed, our methodology is based on load-displacement curves which represent global observables. From a microstructural point of view, the anisotropy would probably also appear in the nucleation, growth and coalescence stages [29]. The approach presented here is however an alternative to obtain better results than with a classical scalar damage approach. The use of a 3D damage tensor with anisotropic damage growth laws would be more accurate, but longer in terms of parameters identification and computation time.

More advanced damage approaches can be found in the literature [42]. Simo and Ju [16, 17] have defined a fourth-order linear transformation operator called the anisotropic damage effect tensor to account for damage anisotropy and grain flow orientation.

\section{Applications}

Different applications are shown here to illustrate the benefit to combine the different improvements presented in the previous section. The first example shows the comparison of strong and weak coupling in terms of CPU time and accuracy. The second and third examples (compression of a tapered specimen and extrusion process) deal with compressive damage evolution. It is shown that combining crack closure effects together with a stress triaxiality cut-off value gives more predictive results. The fourth example illustrates the use of the new Lemaitre damage potential applied to a compression-tension test. Finally, in the last example, the anisotropic methodology based on the computation of grain flow orientation is applied to the simulation of compression of a notched cylindrical specimen.

\section{Weak coupling vs. strong coupling}

Weak coupling and strong coupling have been compared on a tensile axisymmetrical specimen as shown in Fig. 5. A tensile velocity $(\mathrm{v}=0.1 \mathrm{~mm} / \mathrm{s})$ is applied until the displacement equals $0.6 \mathrm{~mm}$. 

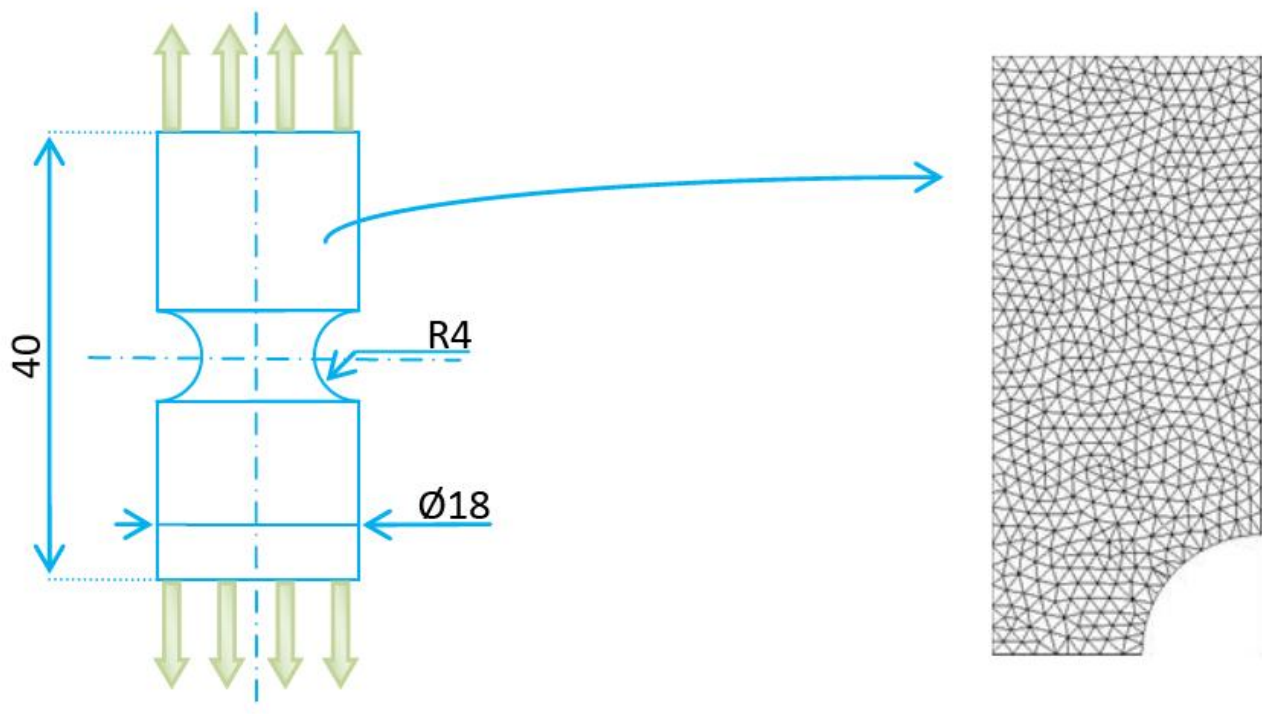

Fig. 5: a) geometry ( $\mathrm{mm})$ of an axisymmetric tensile specimen and b) initial mesh for the axisymmetric simulation.

Table 1 gives the materials and damage parameters used for this study and corresponding to an aluminium alloy [43].

Table 1: Materials and damage parameters for the tensile test

\begin{tabular}{|l|l|l|}
\hline Young modulus & $E$ & $69000 \mathrm{MPa}$ \\
\hline Poisson ratio & $v$ & 0.3 \\
\hline Initial yield stress & $\sigma_{Y}$ & $80.56 \mathrm{MPa}$ \\
\hline Yield stress & $\sigma_{0} \bar{C}_{p}$ & $589\left(0^{-4}+\bar{\varepsilon}_{p}{ }^{\mathrm{D}^{2} 16} \mathrm{MPa}\right.$ \\
\hline Lemaitre exponent parameter & $b$ & 1 \\
\hline Lemaitre denominator parameter & $S_{0}$ & $1.25 \mathrm{MPa}$ \\
\hline Strain threshold & $\bar{\varepsilon}_{D}$ & 0 \\
\hline Critical damage parameter & $D_{c}$ & 1 \\
\hline
\end{tabular}

Weak coupling and strong coupling are compared in terms of equivalent plastic strain (red curves - triangles) and Lemaitre damage values (blue curves - circles) at the centre of the specimen (Fig. 6). The difference observed for both coupling is lower than $4 \%$ while the damage value is lower than 0.4 . When $D$ is in between 0.4 and 0.7 , the error is higher, but remains lower than $10 \%$. Table 2 shows the CPU time obtained for four different mesh sizes and for both coupling methodologies. It can be concluded that weak coupling improves CPU time from $15 \%$ to $30 \%$ without any significant loss of accuracy. As the damage critical value is rarely higher than 0.5 , we have considered that weak coupling could be used for the rest of this study.

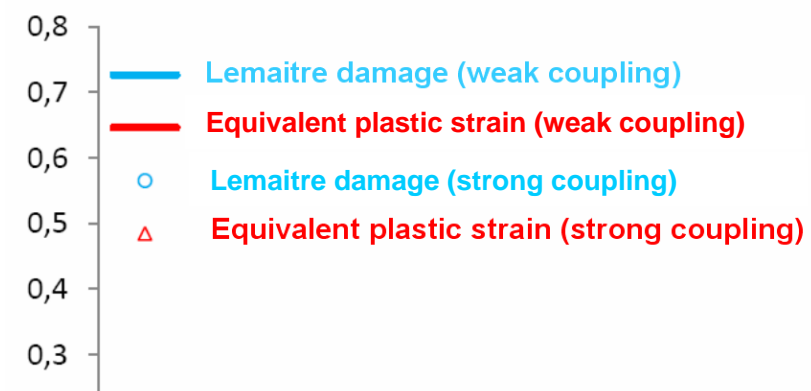


Fig. 6: Comparison of weak coupling and strong coupling in terms of equivalent plastic strain (red) and Lemaitre damage values (blue) at the centre of the tensile specimen.

Table 2: comparison of strong and weak coupling CPU time for different mesh sizes

\begin{tabular}{|c|c|c|c|}
\hline $\begin{array}{c}\text { Mesh size } \\
(\mathbf{m m})\end{array}$ & $\begin{array}{c}\text { Weak coupling } \\
\text { CPU time (s) }\end{array}$ & $\begin{array}{c}\text { Strong coupling } \\
\text { CPU time (s) }\end{array}$ & $\begin{array}{c}\text { Deviation } \\
(\mathbf{\%})\end{array}$ \\
\hline 0.8 & 15 & 19 & 21 \\
\hline 0.4 & 46 & 54 & 15 \\
\hline 0.2 & 66 & 93 & 29 \\
\hline 0.1 & 355 & 468 & 24 \\
\hline
\end{tabular}

\section{Upsetting of a tapered specimen}

In [1] the study of damage growth during the upsetting test of a tapered specimen was presented based on an initial experiment presented in [44]. This test is adapted to quantify materials ductility for bulk metal forming processes. A UNS L52905 lead alloy is used. Table 3 gives the materials properties as well as the damage parameters used for these simulations. These parameters are the ones used in [1]. $\alpha_{f}$ denotes the friction coefficient relative to the Coulomb friction law.

For this mechanical test, fracture occurs either at the centre of the specimen or at the external surface near the equator, depending on materials ductility.

Table 3: Materials and damage parameters for the lead alloy

\begin{tabular}{|l|l|l|}
\hline Young modulus & $E$ & $18000 \mathrm{MPa}$ \\
\hline Poisson ratio & $v$ & 0.4 \\
\hline Initial yield stress & $\sigma_{Y}$ & $43 \mathrm{MPa}$ \\
\hline Yield stress & $\sigma_{0} \bar{\epsilon}_{p}-$ & $66.656 \mathbf{Q} .0133+\bar{\varepsilon}_{p}{ }^{\gamma .1015}$ \\
\hline Coulomb friction coefficient & $\alpha_{f}$ & 0.35 \\
\hline Lemaitre exponent parameter & $b$ & 1 \\
\hline Lemaitre denominator parameter & $S_{0}$ & $1.5 \mathrm{MPa}$ \\
\hline Strain threshold & $\bar{\varepsilon}_{D}$ & 0 \\
\hline Critical damage parameter & $D_{c}$ & 1 \\
\hline Crack closure parameter & $h$ & $0 ; 0.05 ; 0.2 ; 1$ \\
\hline Material damage parameter $(\mathrm{Eq} 20)$ & $\alpha$ & 0 \\
\hline
\end{tabular}


Fig. 7 shows the geometry of the tapered specimen, the boundary conditions as well as the mesh used for this axisymmetric configuration. $\mathrm{P}^{+} / \mathrm{P} 1$ triangular elements are used. An horizontal axis of symmetry is also used. Compression is applied up to a reduction of $65 \%$ of the initial height.

Crack closure effects were accounted for by using the stress decomposition and the $h$ regularization parameter (see equation 14). It was shown in [1] that if $h$ exceeds 0.08 fracture initiation was not predicted correctly. Such a small value would mean that crack closure effects do not have a significant impact on damage growth for this configuration.

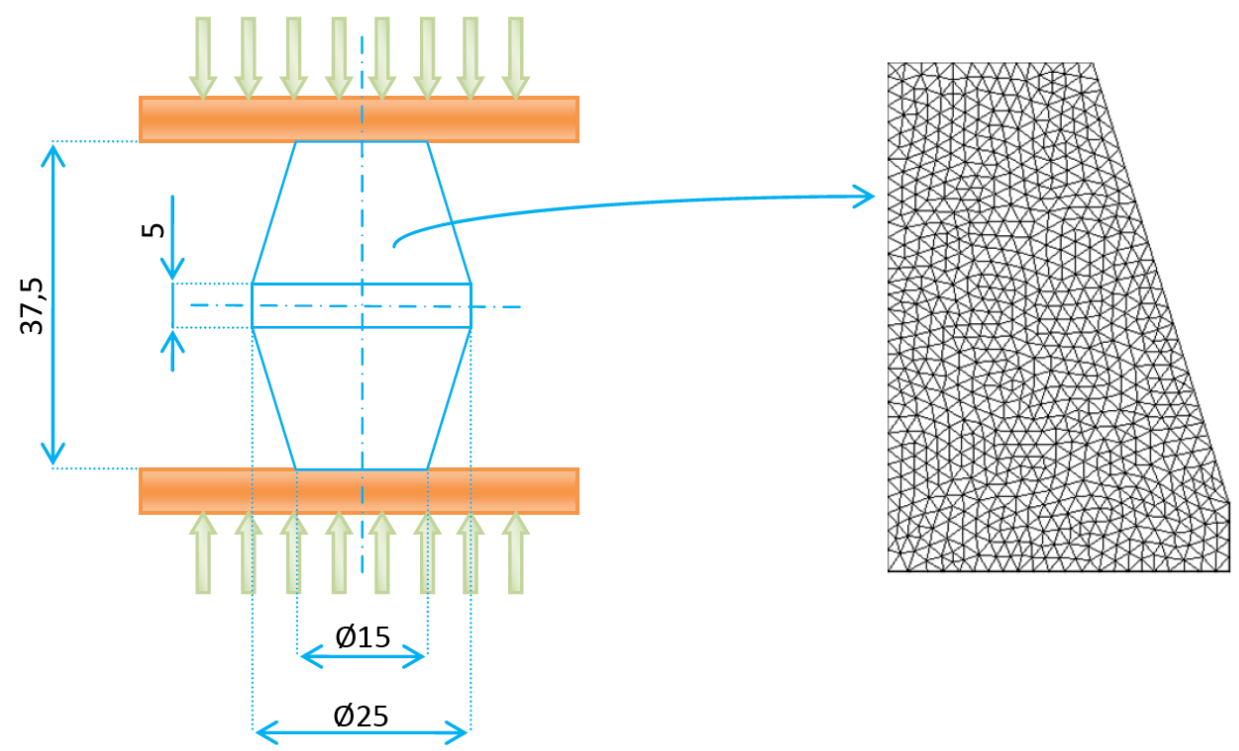

Fig. 7: a) Geometry (mm) of the tapered specimen and b) Initial mesh for the axisymmetric simulation.

In the following, the effect of the stress triaxiality cut-off value is studied. Fig. 8 shows the results obtained without the cut-off value for stress triaxiality for different levels of compression $(25 \%, 45 \%$ and $65 \%)$ and for different values for the $h$ parameter $(1,0.2,0.05$ and 0$)$. The value $h=l$ means that crack closure effects are not accounted for. Damage increase is the localized on the contact area between the tapered specimen and the compression tool, which is not representative to experimental observations. For $h=0.2$, as mentioned by Lemaitre in [14], there is less damage increase in compressive areas. However, the maximal predicted fracture zone is still wrong. For $h=0.05$ damage increases both in the upper contact area and near the equator of the specimen, with a final maximal value obtained in the external equator area, as observed experimentally. Finally, for $h=0$, there is no damage increase in compression, so that damage localizes in the equator area from the beginning. These results are similar to the one obtained in [1]. 


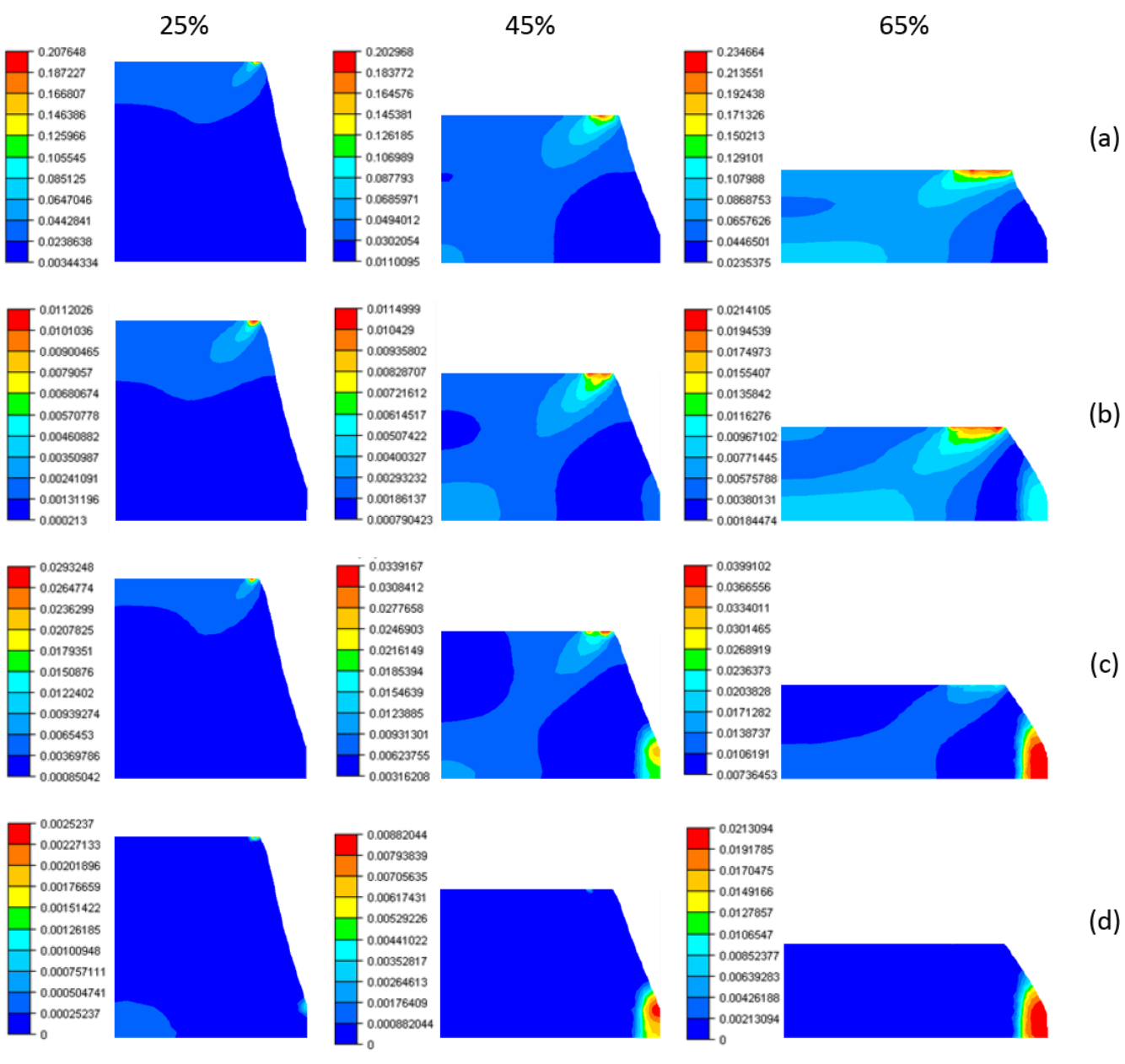

Fig. 8: Lemaitre damage isovalues for different compression rate: (a) $h=1,(b) h=0.2,(c) h=0.05,(d) h=0$.

To study the effect of the stress triaxiality cut-off value, the value of $h$ initially given by Lemaitre has been used $(h=0.2)$. Fig. 9 represents damage values with and without stress triaxiality cut-off for different compression rate. With the stress triaxiality limit, damage increases first in the contact area (25\% of compression rate). But as compression increases, the stress triaxiality in this contact area decreases and becomes lower than $-1 / 3$, so that a new damage concentrated zone appears at the equator of the specimen ( $45 \%$ of deformation). Finally damage concentrates at this external equator location (65\% of deformation) as observed experimentally.

The $h$ value $(h=0.05)$ used by Andrade Pires et al. is very small, and would mean that almost no damage evolution would arise for negative stress triaxiality. With this example, it is shown "qualitatively" that the stress triaxiality cut-off value, coupled with the crack closure effect, enables to obtain physical results with a more "reasonable" $h$ value $(h=0.2)$. 


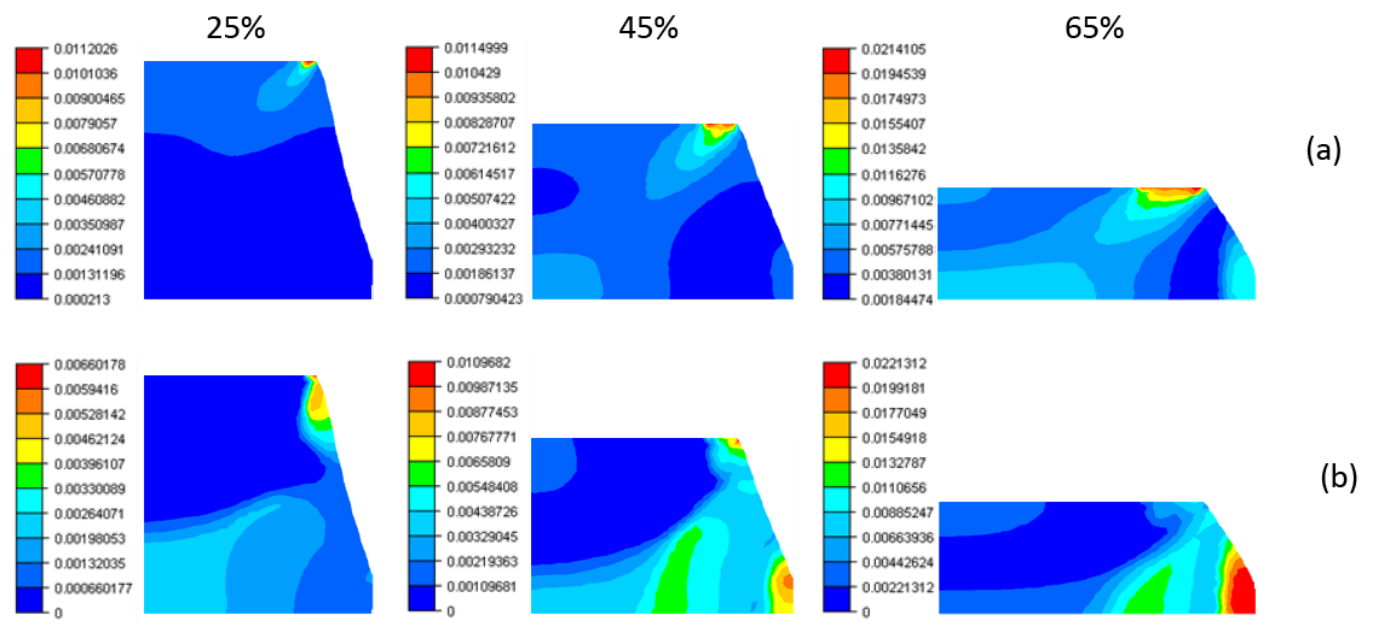

Fig. 9: Lemaitre damage isovalues for different compression rate and with $h=0.2$ : (a) without stress triaxiality limit (b) with stress triaxiality limit.

\section{Application to the extrusion process}

Another classical forming process fracture phenomenon is the central burst (chevrons shape) appearing at the centre of round bars during the extrusion process. The classical Lemaitre damage model is unable to predict these well known chevrons cracking. First a brief description of the process is given. Then the extrusion process is simulated and it is shown how each improvement of the Lemaitre damage model finally enables to account for chevrons cracking.

The extrusion process of round billets enables to reduce the diameter of round bars by pushing a billet into a reduction die (see Fig. 10a). Depending on the reduction ratio, the die angle and friction, central bursts (i.e. in the bulk), called internal chevron cracking (see Fig. 10b), may appear during the process. Unless the products are inspected (using ultrasonic inspection), such internal defects may remain undetected, and later cause failure of the part in use.

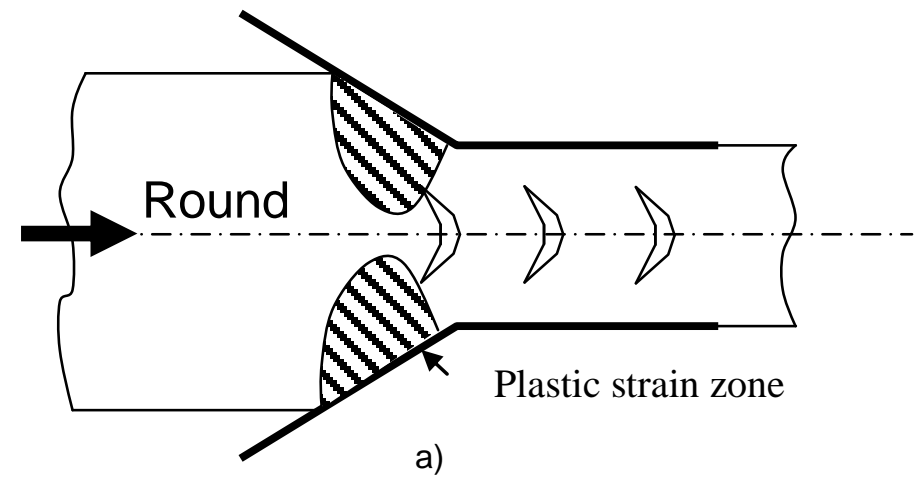

a)

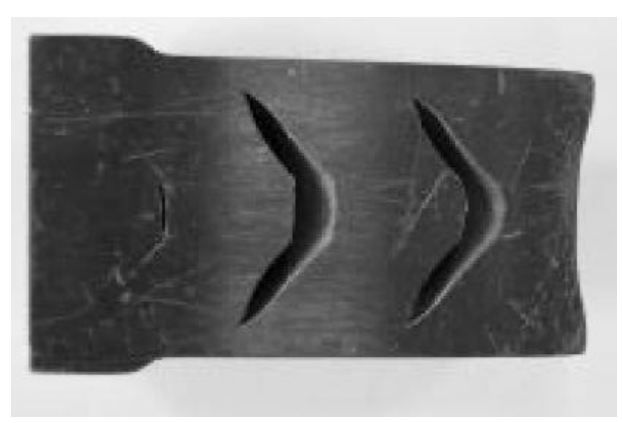

b)

Fig. 10: a) extrusion process - b) internal chevrons cracking.

The axisymmetric version of FORGE2005® is used here. The geometry and tools kinematics are given in Fig. 11. Due to the extrusion process conditions, the simulation can be done using a $2 \mathrm{D}$ axisymmetric configuration. A ductile steel grade is used here with materials and damage parameters given in Table 4 . When damage exceeds the critical value $D_{c}$, the damaged element is deleted from the mesh (kill-element technique). The critical damage value $D_{c}$ can be identified by means of crack initiation during compression test as it is shown in the last example: compression of a cylindrical billet with a longitudinal notch. 


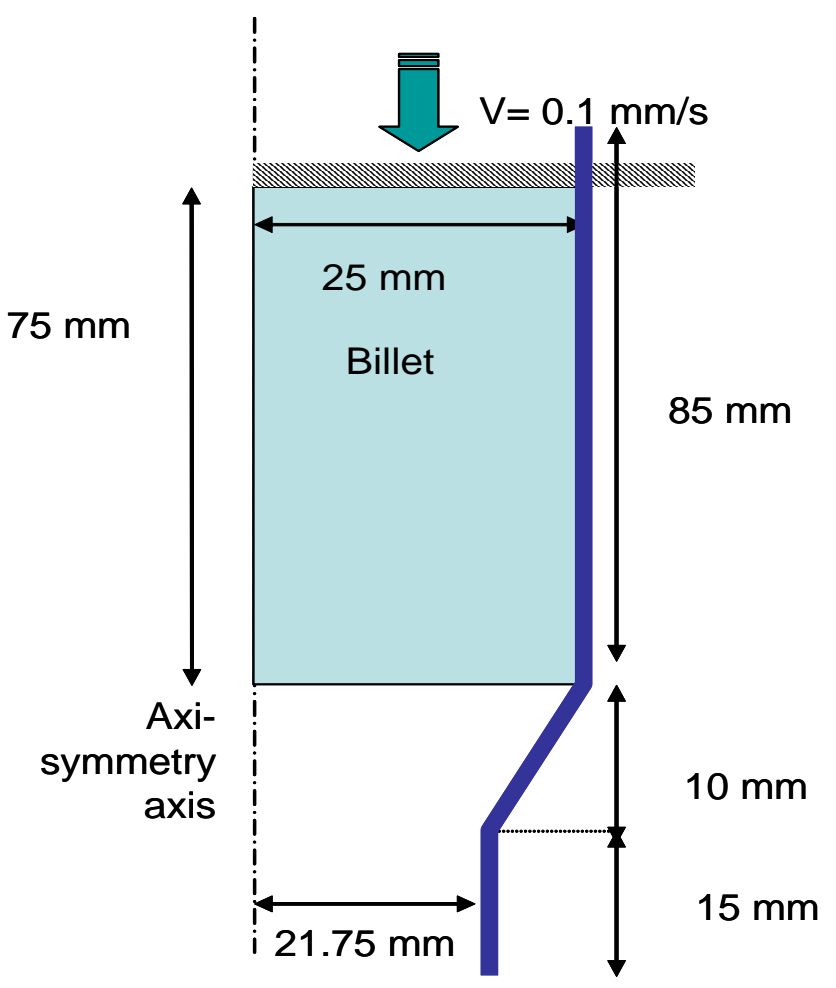

Fig. 11. Extrusion process configuration

First, the Lemaitre coupled damage model has been used without any damage growth for negative stress triaxiality values $(h=0)$. Fig. 12a shows that this model does not predict the good damage location. Fig. 12b represents the evolution of stress triaxiality at a point belonging to the axisymmetric axis. The stress triaxiality ratio is always negative for this configuration, which is the reason why there is no damage increase at the centre of the specimen.

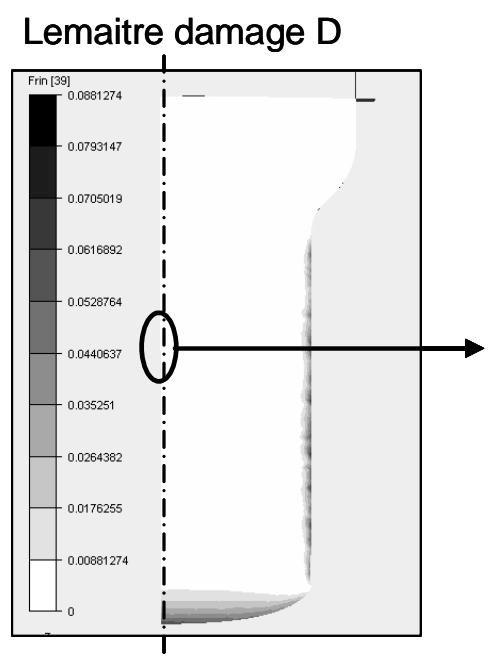

a)

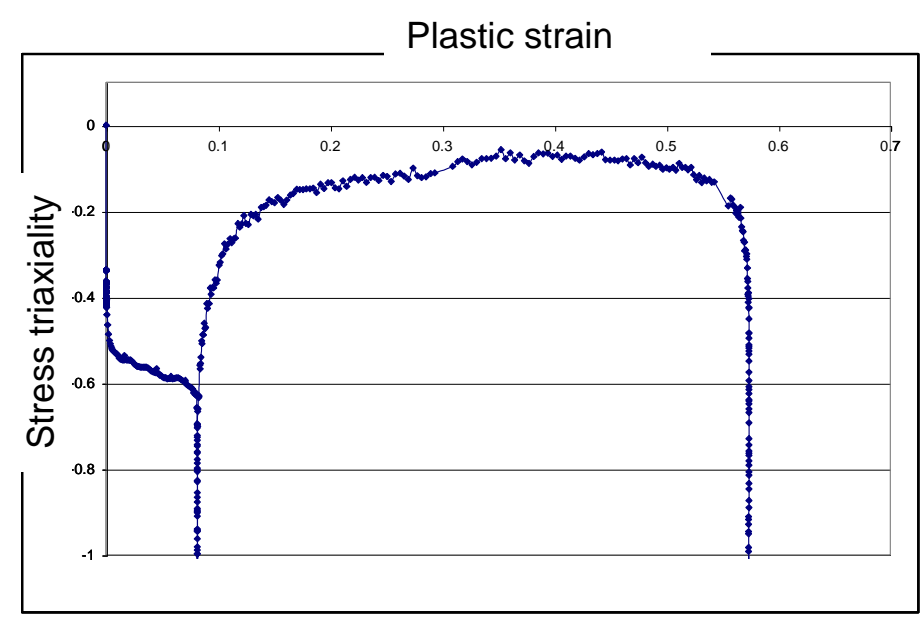

b)

Fig. 12. a) Wrong damage location using the Lemaitre damage model and b) Stress triaxiality vs. plastic strain at the centre of the billet. 
The new features are now added to the model. First, the stress decomposition is used, and damage can increase with negative triaxiality by using the $h$ parameter $(h=0.2)$ as presented in Equation 14. The result presented in Fig. 13a shows that damage is appearing at the centre of the specimen, but that the maximal damage value is reached near the interface, due to the high compressive stress triaxiality resulting from the reduction ratio and from friction. The $-1 / 3$ cut-off value is then used in order to avoid any damage increase when stress triaxiality is lower than $-1 / 3$. Damage is coupled with the mechanical behaviour and the "kill element" technique is used. The location of damage is correct, and the model enables to predict chevrons cracking (Fig. 13b).

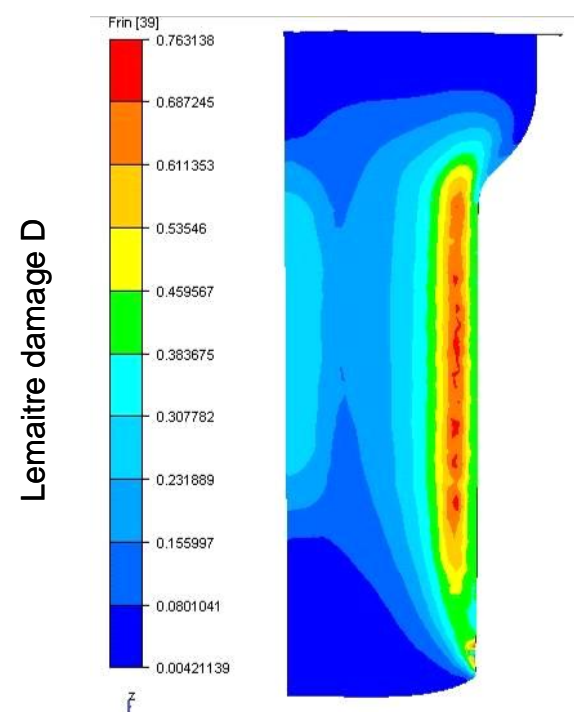

a)

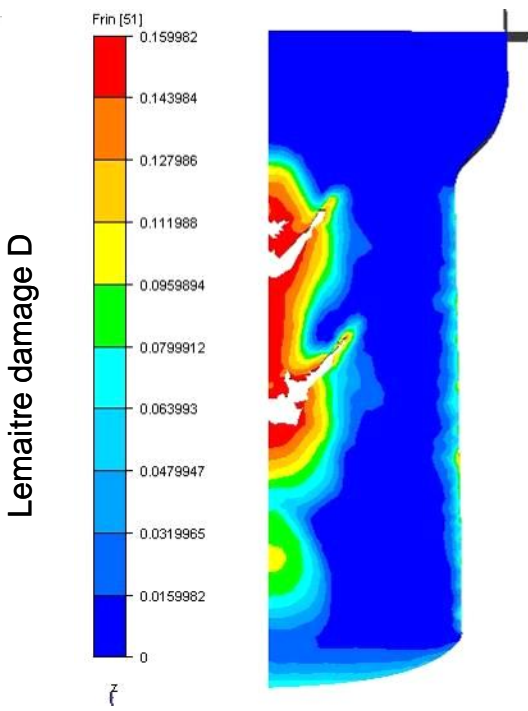

b)

Fig. 13. Chevrons cracking prediction using the extended Lemaitre damage model: a) without the triaxiality cut-off value, b) with the triaxiality cut-off value

\section{Application to precompression-tensile tests}

As presented in Fig. 1, bulk forming processes often exhibit negative stress triaxiality values followed by positive stress triaxiality values and finally leading to fracture. In order to study the influence of pre-compression on fracture, specific compression-tension tests were performed on two ductile steel grades, namely grade A and grade $\mathrm{B}$ (for confidentiality reasons).

Fig. 14 shows the geometry of the diabolo-shaped specimen used for these mechanical tests [45]. In a previous paper [29] the authors have shown that precompression tends to increase ductility for these two ductile steel grades.

An inverse analysis procedure, based on an evolution strategy (ES) methodology, has been used to identify plastic and damage parameters based on precompression-tensile tests. In this paper, we use an enhanced version of ES based on a meta-model. ES methodologies operate on a population of individuals which refers to a specific object parameter set $P i$, its objective function value $F i$, and a strategy parameter $S i$. Index $i$ relates to the number of individuals generated within a population. More details about this ES algorithm can be found in [46].

It has not been possible to match perfectly the experimental results with the classical Lemaitre damage potential for both grades. Fig. 15.a shows that numerical load-displacement curves tend to decrease too much at the end of the tensile part of the test. This leads to an overestimation of the computed damage variable, and to a wrong prediction for fracture initiation. Fig. 15.b presents the load-displacement obtained (after parameters identification) with the new Lemaitre potential presented in this paper. Very good adequacy is found between experimental and numerical results. Table 5 present materials parameters identified for both grades.

The equivalent plastic strain decomposition is important here since $\bar{\varepsilon}_{p}$ is used in the damage potential. If this decomposition was not considered, an overestimation of damage growth would arise. Such decomposition is also 
used by Pirondi and Bonora [41] for low cycle fatigue applications.

For multi-stages bulk forming processes, the material is successively subjected to compression and tension. The use of this damage potential together with the plastic strain decomposition is then more adapted to these forming processes.

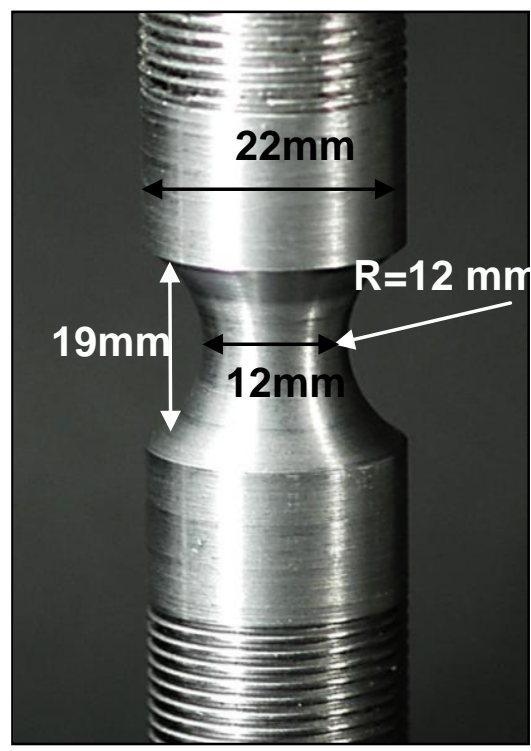

Fig. 14. Geometry of the diabolo-shaped specimen.

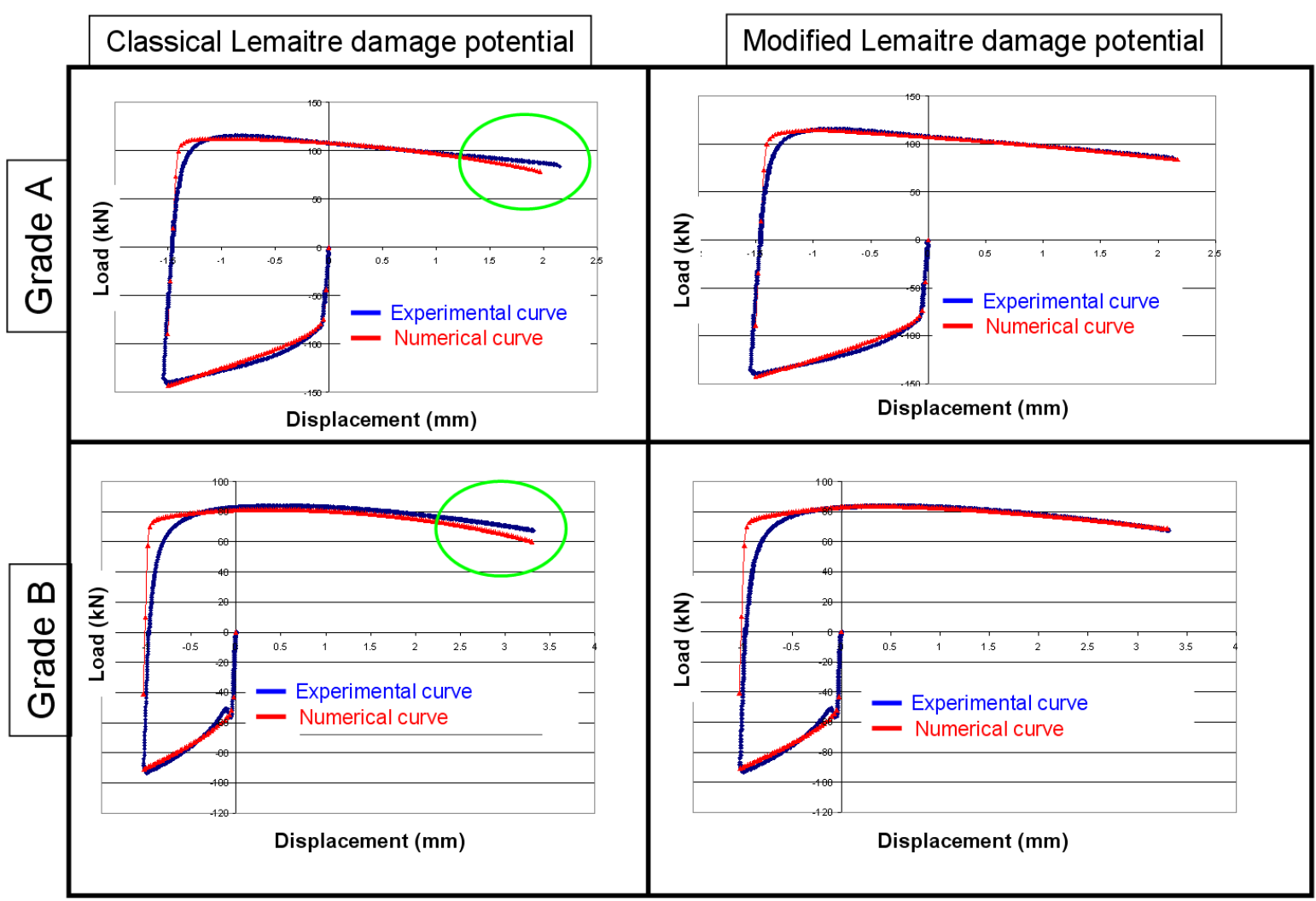

a)

b)

Fig. 15. Comparison between experimental and numerical precompression-tension load displacement curves obtained a) with the Lemaitre damage potential and b) with the modified Lemaitre damage potential. 


\section{Compression of a cylindrical billet with a longitudinal notch}

A cylindrical billet with a longitudinal notch is considered here (Fig. 16). This geometry is interesting for two main reasons:

- fracture is easily localized since it appears at the centre of the notch as the notch opens both in the radial and longitudinal directions during the longitudinal compression;

- mechanical loadings are close to what experiences the material during bulk forming processes. Indeed the plot of stress triaxiality at the centre of the notch is negative at the beginning of the compression test and becomes positive during compression, due to the radial opening of the notch.
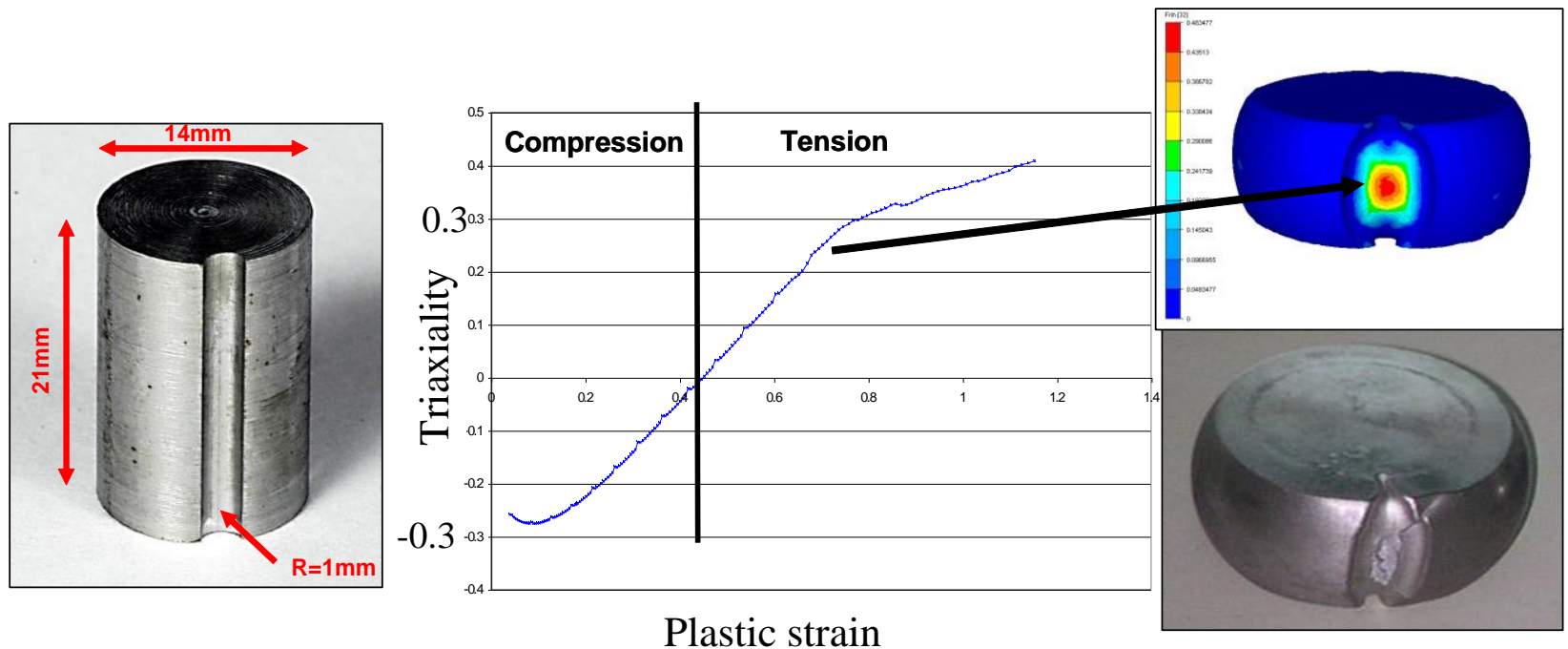

Fig. 16. Notched cylindrical billet subjected to compression - stress triaxiality at the centre of the notch switch from a negative to a positive value.

Material and damage parameters identified previously for Grade A are used here (see Table 5), except for the critical damage value $D_{c}$. As shown in Fig. $2, D_{c}$ depends on the orientation of grain flow orientation with respect to the loading direction. Tensile tests at $0^{\circ}, 45^{\circ}$ and $90^{\circ}$ with respect to the longitudinal direction have thus been used to identify $D_{c}$. As expected, the value of $D_{c}$ decreases while the angle between grain flow orientation and loading direction increases (Table 6).

Table 5: Material parameters identified using inverse analysis for both grades

\begin{tabular}{|c|c|c|c|c|c|c|c|c|c|}
\hline & \multicolumn{4}{|c|}{ Plastic hardening parameters } & \multicolumn{5}{|c|}{ Damage parameters } \\
\hline & $\begin{array}{c}K \\
(M P a)\end{array}$ & $a$ & $n$ & $\beta_{\text {cin }}$ & $\begin{array}{c}S_{0} \\
(M P a)\end{array}$ & $b$ & $\alpha$ & $\varepsilon_{D}$ & $D_{c_{-} 0^{\circ}}$ \\
\hline Grade A & 299 & 1.77 & 0.63 & 0.6 & 40 & 0.79 & 1.32 & 0.070 & 0.50 \\
\hline Grade B & 200 & 2.57 & 0.60 & 0.5 & 35 & 0.75 & 1.52 & 0.145 & 0.40 \\
\hline
\end{tabular}


Table 6: Materials parameters identified using inverse analysis for Grade $A$ and critical damage value for a specimen cut at $0^{\circ}, 4^{\circ}$ and $90^{\circ}$ with respect to grain flow orientation.

\begin{tabular}{|c|c|c|c|c|c|c|c|c|c|c|c|}
\cline { 2 - 12 } & \multicolumn{4}{|c|}{ Plastic hardening parameters } & \multicolumn{1}{c|}{ Damage parameters } \\
\cline { 2 - 11 } & \begin{tabular}{c}
\hline$K$ \\
$(M P a)$
\end{tabular} & $a$ & $n$ & $\beta_{\text {cin }}$ & $\begin{array}{c}S_{0} \\
(M P a)\end{array}$ & $b$ & $\alpha$ & $\varepsilon_{D}$ & $D_{c_{-} 0^{\circ}}$ & $D_{c_{-} 45^{\circ}}$ & $D_{c_{-} 90^{\circ}}$ \\
\hline $\begin{array}{c}\text { Grade } \\
\text { A }\end{array}$ & 299 & 1.77 & 0.63 & 0.6 & 40 & 0.79 & 1.32 & 0.07 & 0.50 & $\mathbf{0 . 3}$ & $\mathbf{0 . 1}$ \\
\hline
\end{tabular}

Experimentally, the displacement leading to fracture initiation ( $\delta_{\exp }^{\text {fract }}$ ) is recorded. Fracture occurs for an height $\mathrm{h}=12.6 \mathrm{~mm}$, so that $\delta_{\exp }^{\text {fract }}=21-12.6=8.4 \mathrm{~mm}$.

The numerical simulation of the compression test is performed. The maximal numerical damage value $D_{\max }$ is noted when the numerical compression displacement reaches $\delta_{\exp }^{\text {fract }}$.

The numerical maximal damage value is $D_{\max }=0.19$.

As the billet has been cut in the longitudinal direction, grain flow orientation is parallel to the axis of compression. Due to the radial opening of the notch during the compression test, the principal stress direction is radial (Fig. 17), so that $\beta=90^{\circ}$. The numerical prediction $\left(D_{\max }=0.19\right)$ is very close to the experimental one $D_{c_{-} 90^{\circ}}=0.18$.

For this particular example the prediction of fracture initiation using this simple anisotropic scalar model is very good.

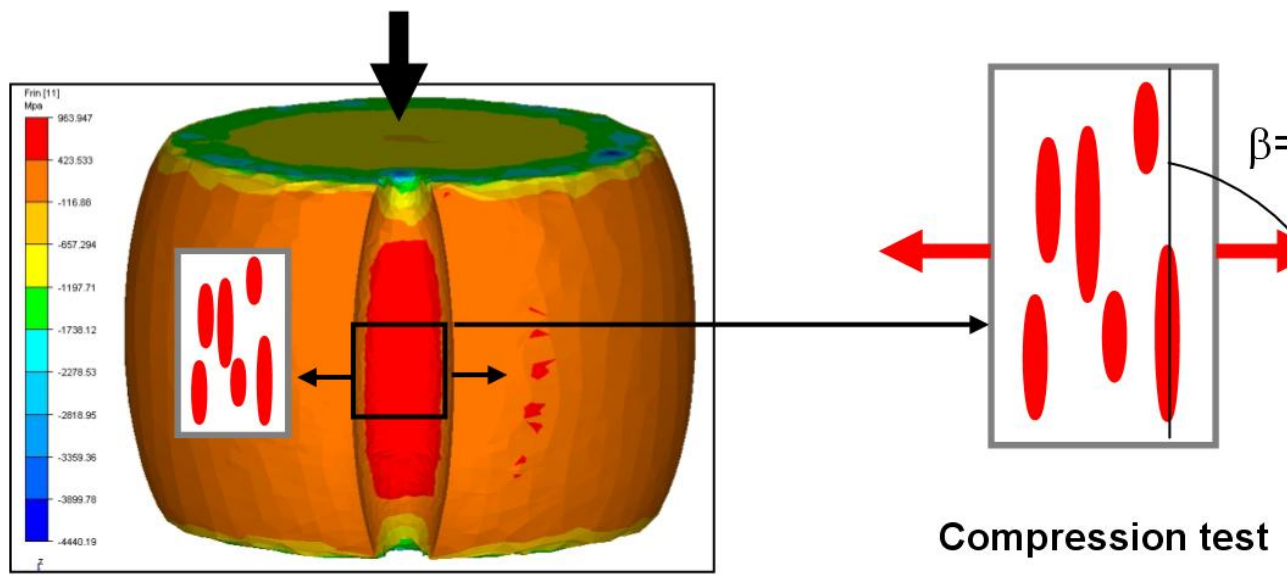

Fig. 17. Orientation of the principal stress field with respect to the orientation of grain flow orientation for the compression test.

\section{Conclusion}

Different extensions of the Lemaitre damage model have been presented to improve damage prediction in forming processes numerical simulations and for anisotropic damage behaviour.

Concerning compressive damage evolution, the stress tensor is split into a positive part and a negative part. Damage growth is modified for the negative part by the use of a $h$ regularization parameter that accounts for crack 
closure effects. Besides, a cut-off value is used in order to avoid any damage growth for stress triaxiality lower than $-1 / 3$. These two techniques were already published in the literature. However, it has been shown here that the combination of both methodologies gives better results in terms of fracture initiation both on a compression test of a tapered specimen and on the prediction of chevrons cracking during the extrusion process.

A new damage potential has also been defined to deal with highly ductile materials. A new term, depending on the equivalent plastic strain, is added. This equivalent plastic strain is split into a negative part and a positive part in order to make the difference between damage growth in compressive stages and in tensile stages. This new Lemaitre damage potential gives good results on pre-compression - tensile tests for Diabolo shape specimens.

For anisotropic damage materials, a phenomenological methodology based on the computation of the grain flow orientation has been presented. In a first approximation, it has been shown that damage anisotropy - for the material studied here (Grade A) - can be handled on the only basis of an anisotropic critical damage value. This critical damage value can be considered as a linear function of the angle formed between the grain flow orientation, and the direction of principal loading. The compression test of a notched cylinder has shown the efficiency of this approach.

However, it must be stated that a full 3D damage approach would be more accurate when dealing with complex non-monotonic loading. The methodology described here is an alternative solution that is easier to apply within an industrial framework.

Acknowledgements. The present study was supported by an industrial consortium regrouping CETIM, Ascometal Creas, Lisi Automotive, Setforge and SNR Roulements. The authors wish to acknowledge the industrial partners for this support.

\section{References}

[1] Andrade Pires FM, César de Sa JMA, Costa Sousa L, Natal Jorge RM (2003) Numerical Modelling of ductile plastic damage in bulk metal forming. Int. J. Mech. Sc. 45:273-294

[2] César de Sa JMA, Areias PMA., Zheng C (2006) Damage modelling in metal forming problems using an implicit non-local gradient model Comp. Meth. Appl. Mech. Eng. 195(48-49):6646-6660

[3] Brunet M, Morestin F, Walter H (2004) Damage Identification for Anisotropic Sheet-Metals Using a Non-

Local Damage Model, Int. J. Damage Mech. 13:35-57

[4] Claire D, Hild F, Roux S (2007) Identification of a Damage Law by Using Full-field Displacement Measurements, Int. J. Damage Mech. 16:179-197

[5] Jirasek M (1998) Nonlocal models for damage and fracture: comparison of approaches. Int. J. Solids Structures 35 (31-32):4133-4145

[6] Steinmann P (1999) Formulation and computation of geometrically non-linear gradient damage. Int. J. Numer. Eng. 46:757-779

[7] Peerlings RHJ, de Borst R, Brekelmans WAM, de Vree JHP (1999) Gradient enhanced damage for quasibrittle materials. Int. J. Numer. Engng. 39:3391-3403

[8] Peerlings RHJ, Geers MGD, de Borst R, Brekelmans WAM (2001) A critical comparison of nonlocal and gradient enhanced softening continua. Int. J. Solids Structures 38:7723-7746

[9] Cockcroft MG, Latham DJ (1968) Ductility and the workability of metals. J. Inst. Met. 96:33-39

[10] McClintock FA (1968) A criterion for ductile fracture by growth of holes. J. Appl. Mech. 35:363-371

[11] Rice JR, Tracey DM (1969) On the ductile enlargement of voids in triaxial stress fields. J. Mech. Phys. Solids. 17:201-217

[12] Oyane M, Sato T, Okimoto K, Shima S (1980) Criteria for ductile fracture and their applications. J. Mech. Work. Tech. 4:65-81

[13] Bai Y, Wierzbicki T (2008) A new model of metal plasticity and fracture with pressure and Lode dependence, Int. J. Plast. 24:1071-1096

[14] Lemaitre JA (1996) A course on damage mechanics. Springer-Verlag

[15] Chaboche JL (1981) Continuous damage mechanics - a tool to describe phenomena before crack initiation. Nuclear Engng. Design. 64:233-247

[16] Simo JC and Ju JW (1987) Strain- and Stress-Based Continuum Damage Models - I. Formulation. Int. J. Solids Structures 23(7):821-840.

[17] Simo JC and Ju JW (1987) Strain- and Stress-Based Continuum Damage Models - II. Computational Aspects. Int. J. Solids Structures 23(7):841-869 
[18] Tvergaard V, Needleman A (1984) Analysis of the cup-cone fracture in a round tensile bar. Acta Metall. Mater. 32:157-169

[19] Gurson AL (1977) Continuum theory of ductile rupture by void nucleation and growth: Part I - Yield criteria and flow rules for porous ductile media. J. Eng. Mat. Tech. 99:2-15

[20] Xue L (2007) Damage accumulation and fracture initiation in uncracked ductile solids subject to triaxial loading, Int. J. Solids Structures 44:5163-5181

[21] Mocellin K, Fourment L, Coupez T, Chenot JL (2001) Toward large scale F.E. computation of hot forging process using iterative solvers, parallel computation and multigrid algorithms. Int. J. Num. Meth. Eng. 52:473-488

[22] Chenot JL, Fourment L, Mocellin K (2002) Numerical treatment of contact and friction in FE simulation of forming processes. J. Mat. Proc. Tech. 125-126(9):45-52

[23] Chenot JL, Massoni E (2006) Finite element modelling and control of new metal forming processes. Int. J. Mach. Tools Mf. 46(11):1194-1200

[24] Chastel Y, Diop A, Fanini S, Bouchard PO, Mocellin K (2008) Finite Element Modeling of Tube Piercing and Creation of a Crack, Int. J. Mater. Form. 1:355-358

[25] Sornin D, Fayolle S, Bouchard PO, Massoni E (2009) Plastic instabilities analysis during T-shaped tubes hydro-forming process, Int. J. Mater. Form. 2:131-144

[26] Saanouni K (2008) On the numerical prediction of the ductile fracture in metal forming. Eng. Fract. Mech. 75(11):3545-3559

[27] Lemaitre J, Desmorat R (2005) Engineering Damage Mechanics, Springer-Verlag

[28] Bao Y, Wierzbicki T (2005) On the cut-off value of negative triaxiality for fracture. Eng. Fract. Mech. $72: 1049-1069$

[29] Bouchard PO, Bourgeon L, Lachapele H, Maire E, Verdu C, Forestier R, Logé R (2008) On the influence of particles distribution and reverse loading on damage mechanisms of ductile steel alloys, Mat. Sci. Eng. A. 496:223-233

[30] Pichelin E, Coupez T (1998) Finite element solution of the 3D mold filling problem for viscous incompressible fluid. Comp. Meth. Appl. Mech. Eng. 163(1-4):359-371

[31] Coupez T, Digonnet H, Ducloux R (2000) Parallel meshing and remeshing. Appl. Math. Model. 25(2):153175.

[32] Besson J. (2004) Local approach to fracture. Les Presses Ecole des Mines de Paris

[33] Ladevèze P (1983) On a anisotropic damage theory. Proceedings of CNRS International Colloquium 351Failure criteria of structured media, Vilars-de-Lans, France, Boehler J. P editor. 355-363

[34] Vaz Jr M, de Santi Jr MN, Verrana GO and de Souza Neto EA (2005) Further studies on assessing ductile fracture using continuous damage coupled to an elasto-plastic material model. COMPLAS 2005.

[35] Bridgman PW (1964) Studies in large plastic flow and fracture. Cambridge, MA: Harvard University Press

[36] Liu DS, Lewandowski JJ (1993) The effects of superimposed hydrostatic pressure on deformation and fracture: part I. Monolithic 6061 aluminium. Metall. Mater. Trans. 24A:601-608

[37] Margevicius RW, Lewandowski JJ (1994) The influence of hydrostatic pressure on fracture of singlecrystal and polycrystalline NiAl. Metall. Mater. Trans. 25A:1457-1470 166

[38] Tai WH, Yang BX (1986) A new microvoid-damage model for ductile fracture. Eng. Fract. Mech. 48:151-

[39] Chandrakanth S, Pandey PC (1995) An isotropic damage model for ductile material. Eng. Fract. Mech. 50:457-465

[40] Bonora N (1997) A nonlinear CDM model for ductile failure. Eng. Fract. Mech. 58:11-28

[41] Pirondi A, Bonora N (2003) Modeling ductile damage under fully reversed cycling. Comp. Mat. Sc. 26:129-141

[42] Voyadjis GZ and Kattan PI (2005) Damage Mechanics. Boca Ratton, CRC Press

[43] Vaz Jr. M and Owen DRJ (2001) Aspects of Ductile fracture and adaptive mesh refinement in damaged elasto-plastic materials, Int. J. Numer. Meth. Eng. 50:29-54

[44] Gouveia BPPA, Rodrigues JMC, Martins PAF (1996) Fracture predicting in bulk metal forming. Int. J. Mech. Sci., 38:361-372

[45] Bao Y and Treitler R (2004) Ductile crack formation on notched Al2024-T351 bars under compressiontension loading, Mat. Sci. Eng. A. 384(1-2):385-394

[46] Peyrot I, Bouchard PO, Ghisleni R and Michler J (2009) Determination of plastic properties of metals by instrumented indentation using a stochastic optimization. J. Mater. Res. 24(3):936-947 